\title{
Static Measurements on HTS Coils of Fully Superconducting AC Electric Machines for Aircraft Electric Propulsion System
}

\author{
Benjamin B. Choi ${ }^{1}$, Keith Hunker ${ }^{2}$, Jason Hartwig ${ }^{3}$, and Gerald V. Brown ${ }^{4}$ \\ National Aeronautics and Space Adminstration, Glenn Research Center, Cleveland, Ohio, 44135
}

\begin{abstract}
The NASA Glenn Research Center (GRC) has been developing the high efficiency and high-power density superconducting (SC) electric machines in full support of electrified aircraft propulsion (EAP) systems for a future electric aircraft. A SC coil test rig has been designed and built to perform static and $\mathrm{AC}$ measurements on BSCCO, $(\mathrm{RE}) \mathrm{BCO}$, and YBCO high temperature superconducting (HTS) wire and coils at liquid nitrogen $\left(\mathrm{LN}_{2}\right)$ temperature. In this paper, DC measurements on five SC coil configurations of various geometry in zero external magnetic field are measured to develop good measurement technique and to determine the critical current $\left(I_{c}\right)$ and the sharpness ( $n$ value) of the superto-normal transition. Also, standard procedures for coil design, fabrication, coil mounting, micro-volt measurement, cryogenic testing, current control, and data acquisition technique were established. Experimentally measured critical currents are compared with theoretical predicted values based on an electric-field criterion $\left(E_{c}\right)$. Data here are essential to quantify the SC electric machine operation limits where the SC begins to exhibit non-zero resistance. All test data will be utilized to assess the feasibility of using HTS coils for the fully superconducting AC electric machine development for an aircraft electric propulsion system.
\end{abstract}

\section{Introduction}

$\mathrm{T}$ he NASA Glenn Research Center (GRC), in collaboration with the aerospace industry and academia, has been developing technologies to make large reductions in aircraft energy use, emissions, and noise by 2040 . One concept is a future electric aircraft employing a Turbo-electric Distributed Propulsion (TeDP) system in full support of Electrified Aircraft Propulsion (EAP) system. Highly distributed propulsion offers great aerodynamic design potential, yet each individual electric machine adds to the overall system inefficiency. A fully superconducting machine has been developing at GRC (see Fig. 1), and it offers potential for specific powers up to $40 \mathrm{~W} / \mathrm{g}$ and $99 \%$ efficiency, if alternating current (AC) losses can be minimized. The ultimate goal of the SC AC electric machines is to demonstrate that intermediate temperature superconducting coils can be designed and fabricated for use near $20 \mathrm{~K}$ in AC electromagnetic circuits with electrical losses sufficiently low in order to prevent localized quenching that causes the sudden loss of superconductivity when the its temperature rises above the superconductivity threshold. Thus, both SC wire fabrication and fundamental knowledge have been invested, and in-house expertise and NASA contracts for coil prototype fabrication have been utilized. Further, previous successful design concepts for cryogenic coil testing will be leveraged.

For the SC electric machine operating point of view, it is essential to quantify its operation limits where the SC behaves as a normal conductor and thus begins to exhibit non-zero resistance. Thus, this paper presents design, fabrication, and test results for the recently conducted high temperature superconducting (HTS) Bismuth Strontium Calcium Copper Oxide (BSCCO), Rare Earth Barium Copper Oxide (REBCO), and Yttrium Barium Copper Oxide (YBCO) coil tests in liquid nitrogen $\left(\mathrm{LN}_{2}\right)$. The purpose of these tests is to obtain knowledge on how to fabricate and test several different sized HTS coil prototypes and to obtain performance data at cryogenic temperatures as it relates to technology development for air breathing EAP system, such as fixed wing and vertical lift aircrafts. Five HTS coils of varying wire bending diameter, thickness and length were submerged in $\mathrm{LN}_{2}$ to obtain electrical performance characteristics at $77 \mathrm{~K}$. Voltage, induced magnetic field, resistance, and coil temperature were

\footnotetext{
${ }^{1}$ Aerospace Engineer, LMR, Materials and Structures Division, MS 23/3.

${ }^{2}$ Aerospace Engineer, LED, Power Division, MS 309/2.

${ }^{3}$ Aerospace Engineer, LTF, Propulsion Division, MS 86/12, and Senior Member.

${ }^{4}$ Senior Research Engineer, LMR, Materials and Structures Division, MS 49/8.
} 
measured as a function of current. Controlled $N$-point current ramps were conducted across the range of $0<\mathrm{I}<120$ $\mathrm{A}$ at DC level in zero external magnetic field, and the experimental data were collected for both ramping up and down current. Superconducting operation limits were also quantified by determining the critical current at which the superconductor coil began to exhibit non-zero resistance. Established knowledges at $77 \mathrm{~K}$ here such as lessons learned, data analysis skills, standard procedures for coil design, fabrication, and measurement technique will be used to assess the feasibility of using HTS wire materials in future cryogenically-cooled fully superconducting EAP systems. Also, it would be a guide for the low temperature $(20 \mathrm{~K})$ superconducting coil $\left(\mathrm{MgB}_{2}\right)$ test bed, which is under development at GRC.

\section{GRC Superconducting Motor and HTS Coil Test Setup}
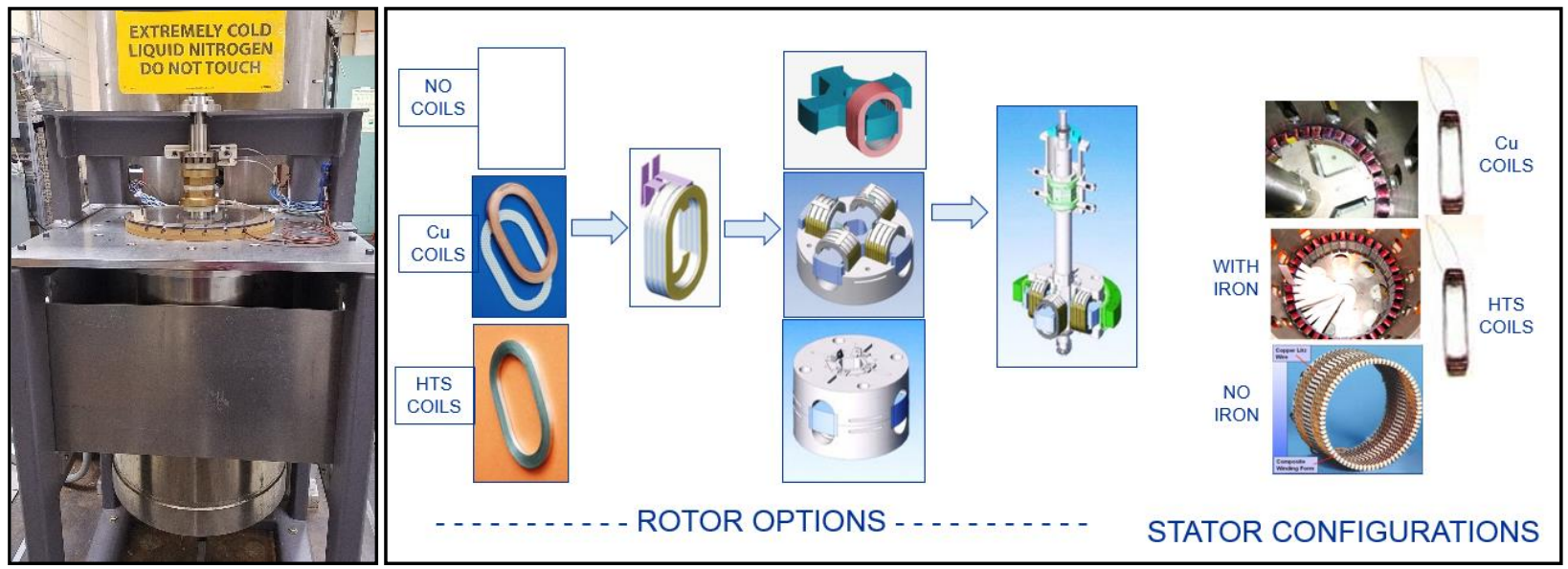

Figure 1. High efficiency and high-power density superconducting motor and its schematic drawing.

Figure 1 illustrates the GRC's fully superconducting motor and its schematic drawing that has been developed for achieving high efficiency (99\%) and high-power density (up to $40 \mathrm{~W} / \mathrm{g}$ ) in full support of NASA mission-critical EAP system for a future electric aircraft. The rotor has four poles and the options of using no coils for the reluctance motor operation, or of using either "racetrack" copper or HTS coils for the synchronous wound rotor operation. The stator has thirty-six teeth and the configuration of option to use either the copper coils or HTS coils as well as the ironless motor operation. For the stator coil and ironless motor coil, due to the minimum bending diameter limitation only REBCO coils were fabricated and tested. Thus, a variety of rotor options and stator configurations can be obtained and summarized in Table 1.

Table 1: Five operation modes for the superconducting motor.

\begin{tabular}{|l|c|c|c|}
\hline & & Rotor Options & Stator Configurations \\
\hline $\mathbf{1}^{\text {st }}$ Mode & & No Coil & Copper Coils \\
\hline $\mathbf{2}^{\text {nd }}$ Mode & & Copper Coils & Copper Coils \\
\hline $\mathbf{3}^{\text {rd }}$ Mode & & HTS Coils & Copper Coils \\
\hline $\mathbf{4}^{\text {th }}$ Mode & & HTS Coils & HTS Coils \\
\hline $\mathbf{5}^{\text {th }}$ Mode & & REBCO Coils & No Iron \\
\hline
\end{tabular}

A standard superconductor resistance measurement test setup with the combination of Keithley 181 nanovoltmeter and HP 6031A power supply/amplifier instrument is used for measuring the applied current, induced voltage, and resistance. The HP 6031A sourcemeter instrument can source DC currents up to $120 \mathrm{~A}$, which is high enough that a significant change in resistance can be detected at liquid nitrogen temperature $(77 \mathrm{~K})$ in the presence of self-field.

For the sake of simplicity, manual data recording was used first. In this method, the current is increased in steps, and the voltage and current readings were manually recorded after a settling time delay of up to 10 secs. A computer controlled data acquisition system was also developed to record automatically the applied current and induced 
voltage readings as well as the thermal couple signals attached to the HTS coil. The current power supply is remotely controlled with the dSPACE-based control/acquisition system, and the current is increased in steps. A sample time of $0.1 \mathrm{sec}$ is used and the computer averages 30 readings, which are taken over a period of 3 secs, before recording the data after each current increment. It is also capable of calculating the theoretical HTS resistance value to compare with the measured resistance value in real time.

\section{Superconductor Critical Current Measurements and Data Analysis}

The goal of this section is to determine the critical current, $I_{c}$, from voltage vs. current $(V-I)$ curves. Currently, there are several methods of theoretically defining the critical current such as electric-field criterion, resistivity criterion, and offset criterion ${ }^{1-4}$. Each method has its own advantages and disadvantages. However, the electric-field criterion $\left(E_{c}\right)$ is selected here for the data analysis because it is the most used, it is well understood, and it facilitates intercomparing of data ${ }^{1}$. The critical current data analysis based on the electric-field criterion $\left(E_{c}\right)$ was compared to theoretical prediction.

\section{A. BSCCO Rotor Coil}

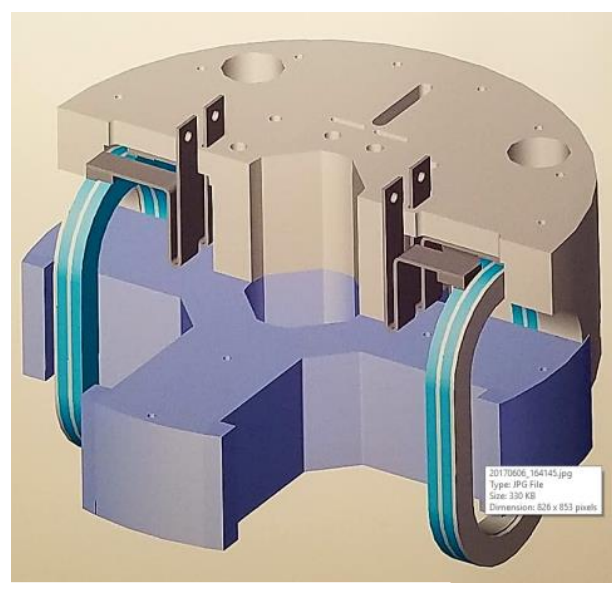

(a)

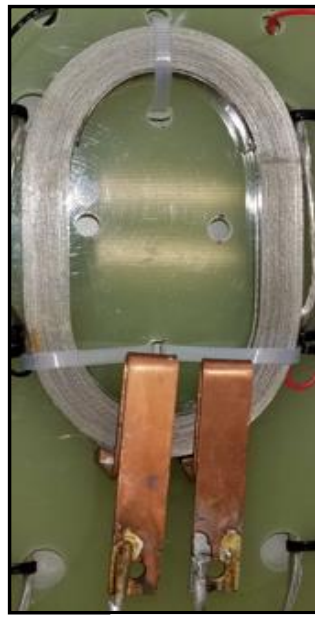

(b)

Figure 2. BSCCO rotor coil sitting on (a) the rotor pole and (b) G10 test fixture.

Compact BSCCO "race-track" prototype of HTS rotor coil-pack (two interconnected coils) has been developed to be installed in the superconducting motor rig and prepared for operation in $\mathrm{LN}_{2}$ as shown in Figure 2. A unique method of fabricating and interconnecting epoxy-bonded coils also has been developed under the in-house research. The BSCCO tape manufactured by American Superconductor is a multifilament composite material with superconductor filaments imbedded in silver and then laminated in thin stainless steel. It has $0.22 \mathrm{~mm}$ in thickness and $4.95 \mathrm{~mm}$ in width. As illustrated in Figure 2, two identical coils are inner-connected by several superconducting junction patches. Each coil has 35 turns and $10.1 \mathrm{~m}$ in length with the electric-field criterion $\left(E_{c}\right)$ of $1 \mu \mathrm{V} / \mathrm{cm}$ at $77 \mathrm{~K}$ in its self-field. Detailed electrical characteristic data are summarized in Table 2.

Table 2: Dimension and technical data for the BSCCO rotor coil.

\begin{tabular}{|l|l|l|l|l|l|l|l|}
\hline Manufacturer & Wire type & Turns/coil & O.D. & I.D. & Length/coil & Measured $\boldsymbol{B} / \boldsymbol{A}$ & $\boldsymbol{L}$ and $\boldsymbol{R} @ \mathbf{4 0 0} \mathrm{Hz}$ \\
\hline AMSC & BSCCO & 35 & $137.2 \mathrm{~mm}$ & $101.6 \mathrm{~mm}$ & $10.1 \mathrm{~m}$ & $10.0 \mathrm{Gauss}$ & $152.2 \mu \mathrm{H}, 397 \mathrm{~m} \Omega$ \\
\hline
\end{tabular}

Figure 3 shows the $V-I$ curves of the top rotor coil for a typical cycle for current ramping up and ramping down, and also shows the corresponding power fitting curve for obtaining the $n$ value. At low transport current up to $52 \mathrm{~A}$, the superconductor has effectively zero resistance, but in the vicinity of critical current $I_{c}$, the voltage rapidly rises as the superconductor reverts to the normal resistive state. The power fitting curve of $y=A+B x^{n}$ was obtained where $A=0.01, B=1.31 \mathrm{E}-28$, and $n=16.47$. Two more cycles were performed and the repeatability between cycles was within a negligible percentage. Data were taken during current ramp down to ensure that there was negligible hysteresis.

Analytical estimation method of defining the critical current is considered first, and its result is compared to the critical-current data based on the electric-field criterion $\left(E_{c}\right)$. 
Figure 4.a shows the low-field range at $77 \mathrm{~K}$ for the field perpendicular to the tape plane ${ }^{5}$. Both multifilamentary composites (MFC)-BSCCO and coated conductor composites (CCC)-YBCO show a rapid dropoff in critical current with increasing field, though CCC is less steep and the tail persists to much higher fields. Note that as in MFC-BSCCO tapes ${ }^{6}$, the CCC is expected to show self-field effects in this temperature and field range, which will cause these curves to shift depending on the total current level. By using a handheld gauss meter with probe, the maximum magnetic field on the rotor coil surface was measured as $1.0 \mathrm{mT} / \mathrm{A}$ in self-field, which means that at $143 \mathrm{~A}$, provided by manufacturer's data sheet, the field would be $0.143 \mathrm{~T}$. Then an operating line (the red dashed line) can be drawn from $(0,0)$ to $(0.143$, 1.0). Here, notice that the graph is normalized so that 1.0 means $100 \mathrm{~A}$. The intersecting point of the red dashed line with the dotted BSCCO critical current point set gives the expected limit of superconductivity as $(0.075,0.5)$. Therefore, the theoretical analysis value of $I_{c}$ is approximated by $143 \mathrm{~A}$ x $0.5 \approx 72 \mathrm{~A}$.

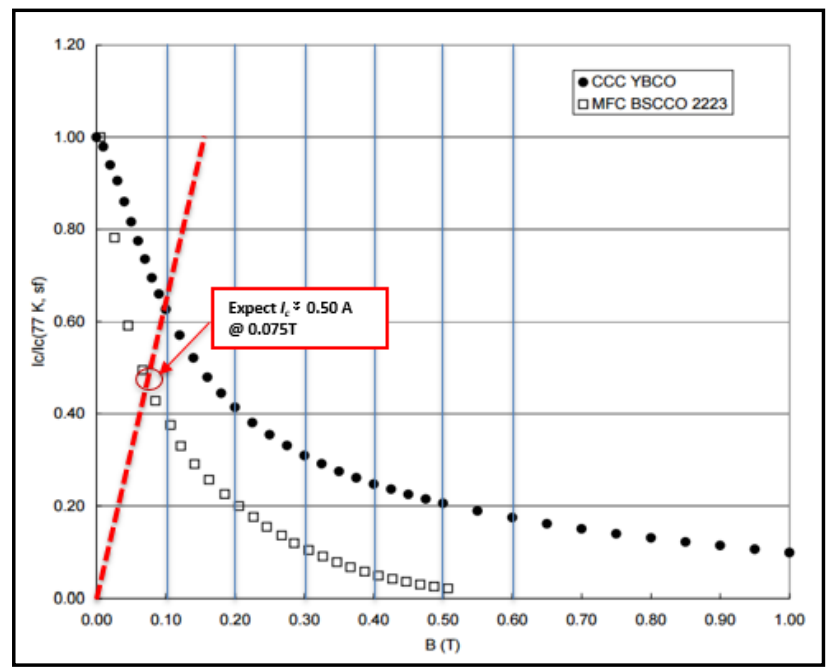

(a)

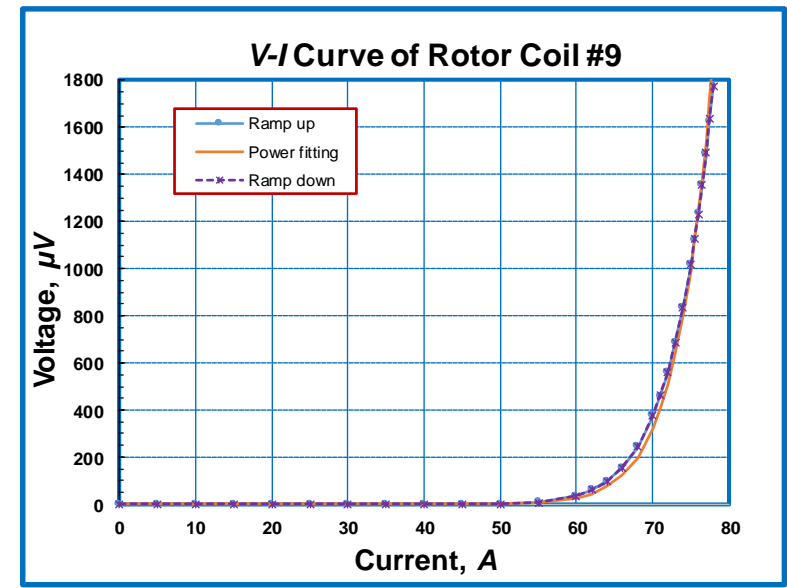

Figure 3. Plots of voltage vs. current for the BSCCO rotor coil and corresponding power fitting curve.

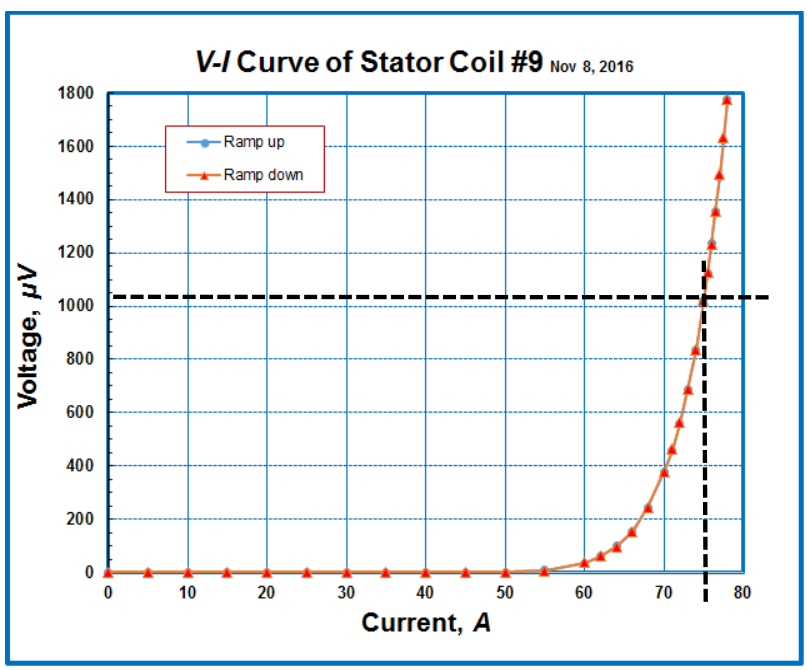

(b)

Figure 4. Critical current analyses using (a) analytical estimation and (b) electric-field criterion.

Now, the critical current analysis based on the electric-field criterion is considered. This criterion facilitates interlaboratory comparisons of data and makes the data useful for engineering design purposes ${ }^{1,2,6}$. The voltage generated depends on the length of superconductor between two voltage taps, so the critical level, $E_{c}$, is usually expressed as an electric field along the conductor. For the HTS coils, it is defined as $E_{c}=1 \mu \mathrm{V} / \mathrm{cm}$. Since the total coil length between the two voltage taps is $10.1 \mathrm{~m}$, the critical voltage level of $1010 \mu \mathrm{V}$ is chosen. As shown in Figure 4.b, the critical current of $\approx 74.5 \mathrm{~A}$ is defined as the current at which the $V-I$ curve intersects with the criterion line (black dashed line). This experimental value of $\approx 74.5 \mathrm{~A}$ is relatively close to the theoretical estimation of $\approx 72 \mathrm{~A}$.

\section{B. YBCO Rotor Coil}

Compact YBCO "race-track" prototype of the rotor coil pack (two interconnected coils) has been also developed to be installed into the superconducting motor rig and prepared for $\mathrm{LN}_{2}$ operation. However, fabricating and interconnecting epoxy-bonded coils of YBCO HTS needed to be adapted from the previously developed method for 


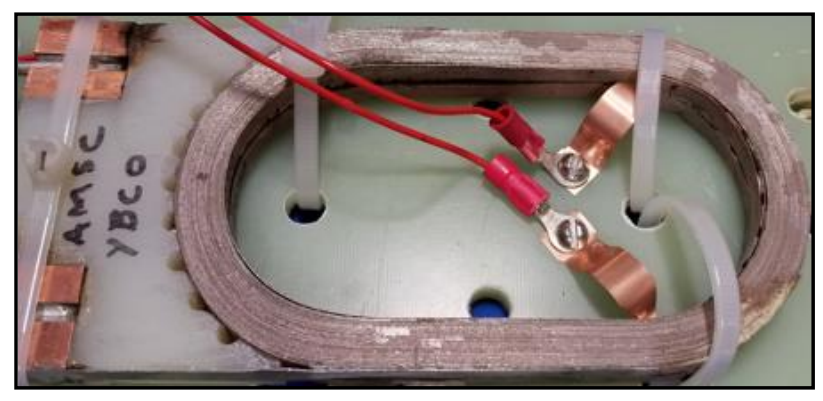

Figure 5. YBCO rotor coil.

the BSCCO material. Because of the different material characteristics and configuration, the mechanical properties of the YBCO tapes are different from those of the BSCCO tape, and required new procedures and fixturing for coil winding and interconnecting. The YBCO tape is manufactured by American Superconductor, and has multilayer laminations with up to 9 thin bonded layers. It has $0.22 \mathrm{~mm}$ in thickness and $4.95 \mathrm{~mm}$ in width. As illustrated in Figure 5, two identical coils are inner-connected by several YBCO junction patches. Each coil has 35 turns and $10.1 \mathrm{~m}$ in length with the electric-field criterion $\left(E_{c}\right)$ of $1 \mu \mathrm{V} / \mathrm{cm}$ at $77 \mathrm{~K}$ in its self-field. Detailed electrical characteristic data are summarized in Table 3.

Figure 6 shows the $V-I$ curves of the top coil, bottom coil, junction patches, and whole coils for a typical ramp up and down. The $V-I$ curve of the whole coils (top red curve) includes the resistance line (slope of $4.6 \mu \Omega$ ) of SC junction patches (brown line), top coil's curve (beige color), and bottom coil's curve (green color). Unlike the BSCCO case, however, all V-I curves have characteristics with sloped baselines. These sloped baselines usually arise in short-coil testing of superconductors with a resistive matrix where the separation between the current contact and voltage tap is small ${ }^{1}$. The best all-around technique to accomplish this is the back-extrapolation method $^{1}$, which requires acquiring $V-I$ data to high voltages. However, in the current case, high-voltage data were obtained already, so the simple baseline method, where the sloped baselines (resistances) are subtracted from the $V-I$ curves in order to extract the intrinsic $V-I$ characteristics, is utilized here. With such alterations of the $V-I$ curves, the corrected $V-I$ curves were obtained as illustrated in Figure 7.b. Note that the bottom coil's $V-I$ curve has not been changed because its original $V$ - $I$ curve is good.

Table 3: Dimension and technical data for the double-layer YBCO rotor coil.

\begin{tabular}{|l|l|l|l|c|l|l|l|}
\hline Manufacturer & Wire type & Turns/coil & O.D. & I.D. & Length/coil & Measured $\boldsymbol{B} / \boldsymbol{A}$ & $\boldsymbol{L}$ and $\boldsymbol{R} @ \mathbf{4 0 0 ~ H z}$ \\
\hline AMSC & YBCO & 35 & $137.2 \mathrm{~mm}$ & $101.6 \mathrm{~mm}$ & $10.1 \mathrm{~m}$ & $20.1 \mathrm{Gauss}$ & $619.4 \mu \mathrm{H}, 1.05 \Omega$ \\
\hline
\end{tabular}

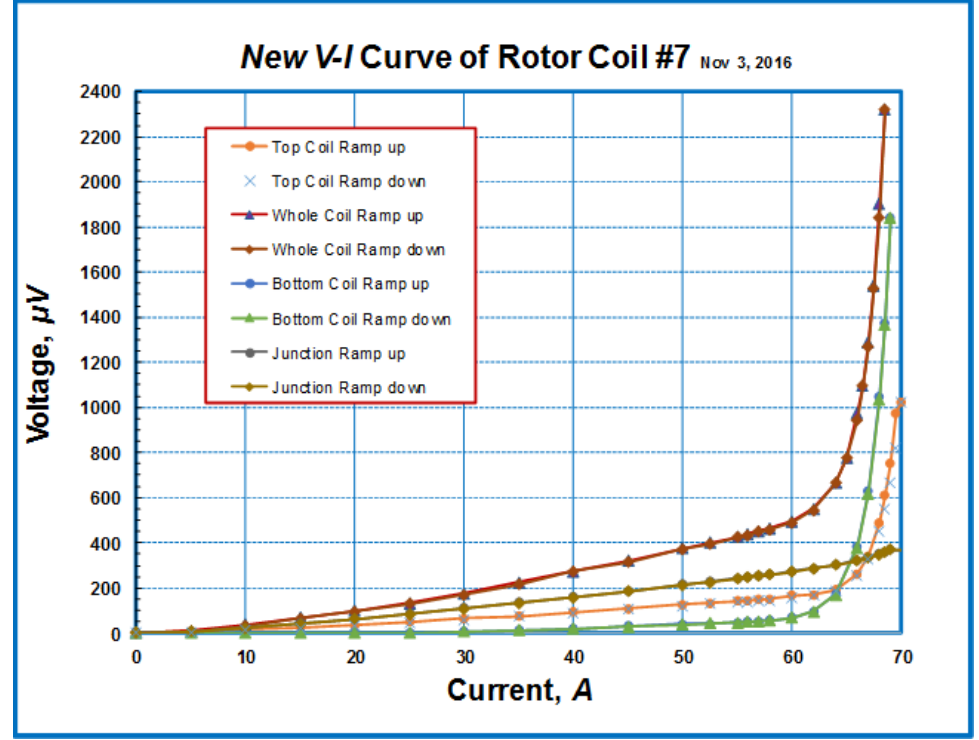

Figure 6. Plots of voltage vs. current for the YBCO rotor coil, showing the $V$ - $I$ curves affected by current-transfer voltage at low-current levels.
Similar to the previous analysis, the maximum magnetic field on the YBCO rotor coil surface was measured as $2.0 \mathrm{mT} / \mathrm{A}$ in self-field, which means that at $132 \mathrm{~A}$, provided by manufacturer's data sheet, the field would be 0.264 T. As shown in Figure 7.a, the operating line (the red dashed line) can be drawn from $(0,0)$ to $(0.264,1.0)$. Here, note that the graph is normalized so that 1.0 means $100 \mathrm{~A}$. The intersecting point of the red dashed line with the dotted YBCO critical current point set gives the expected limit of superconductivity as $(0.125,0.52)$. Therefore, the theoretical analysis value of $I_{c}$ is approximated by $132 \mathrm{~A} \times 0.52 \approx 68.6 \mathrm{~A}$.

Like the previous analysis done in the BSCCO coil, the electric-field criterion is defined as $E_{c}=1 \mu \mathrm{V} / \mathrm{cm}$, and since the total coil length between the two voltage taps is $10.1 \mathrm{~m}$, the critical voltage level of $1010 \mu \mathrm{V}$ 
is chosen. As shown in Figure 7.b, the critical currents of $68 \mathrm{~A}, 70.5 \mathrm{~A}$, and $67 \mathrm{~A}$ are obtained as the $I_{c_{-}} b o t, I_{c_{-}}$top, and $I_{c_{-}}$whole, respectively, where $I_{c_{-}} b o t$ denotes the critical current for bottom coil; $I_{c_{-}}$top for top coil; and $I_{c_{-}}$whole for whole coils. These experimental values of $67 \mathrm{~A}-70.5 \mathrm{~A}$ agree well with the theoretical estimation of $\approx 68.6 \mathrm{~A}$.

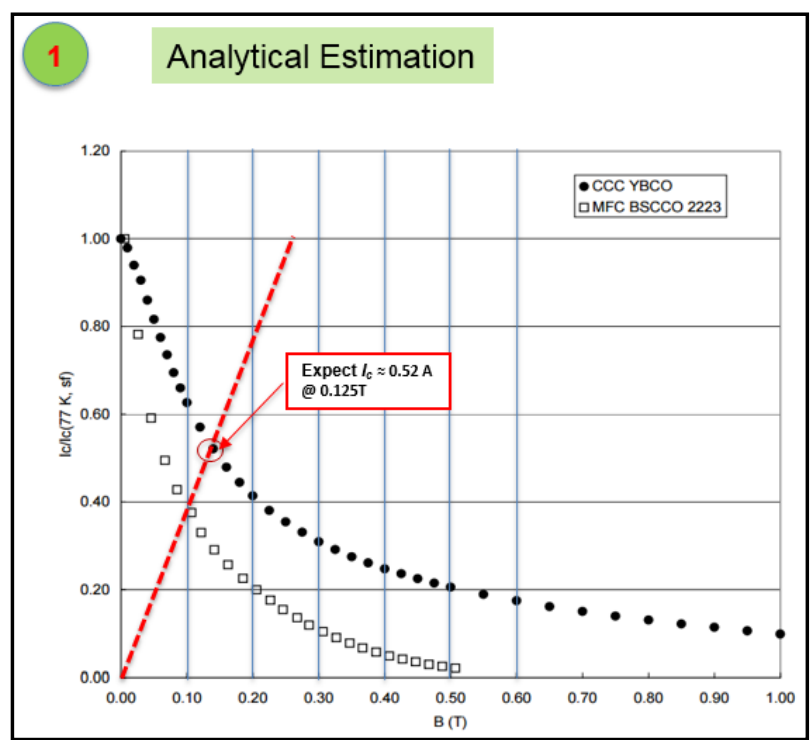

(a)

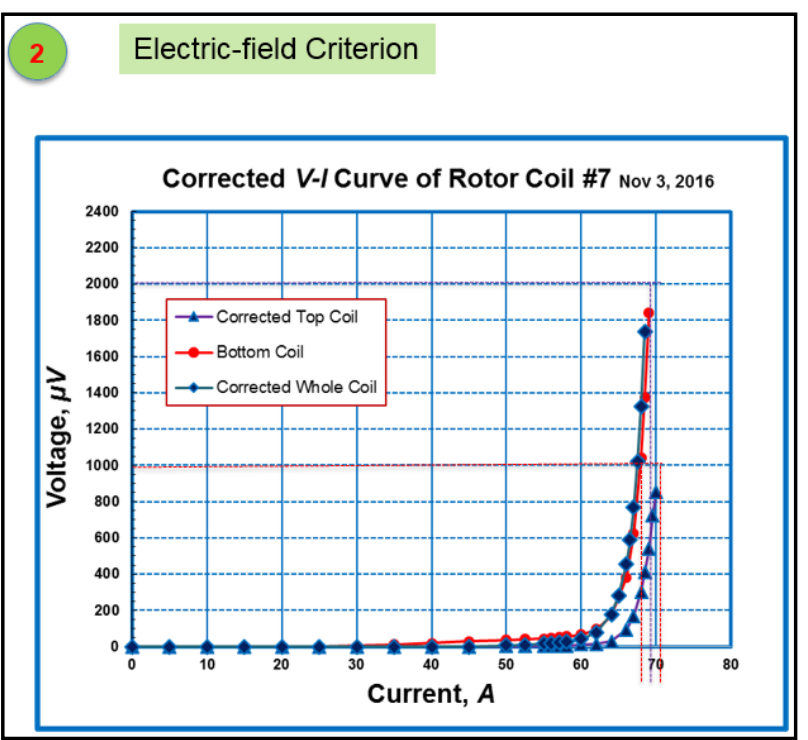

(b)

Figure 7. Critical current analyses of the double-layer YBCO rotor coil using (a) analytical estimation and (b) electric-field criterion.

\section{REBCO Rotor Coil}

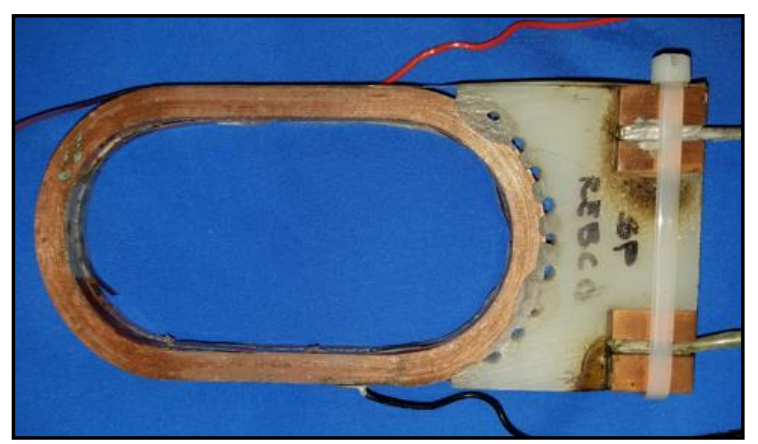

Figure 8. REBCO rotor coil.
In this section, the third candidate of compact "race-track" prototype of the REBCO rotor coil-pack (two interconnected coils) was tested. Fabricating and interconnecting epoxybonded coils of REBCO is the same as those of YBCO coil. The REBCO tape is manufactured by SuperPower and has $0.1 \mathrm{~mm}$ in thickness and $4.0 \mathrm{~mm}$ in width. As illustrated in Figure 8, two identical coils are inner-connected by several superconductor junction patches. Each coil has 35 turns and $10.1 \mathrm{~m}$ in length with the electric-field criterion $\left(E_{c}\right)$ of 1 $\mu \mathrm{V} / \mathrm{cm}$ at $77 \mathrm{~K}$ in its self-field. Detailed electrical characteristic data are summarized in Table 4.

Table 4: Dimension and technical data for the REBCO rotor coil.

\begin{tabular}{|l|l|l|c|c|l|l|l|}
\hline Manufacturer & Wire type & Turns/coil & O.D. & I.D. & Length/coil & Measured $\boldsymbol{B} / \boldsymbol{A}$ & $\boldsymbol{L}$ and $\boldsymbol{R} @ \mathbf{4 0 0} \mathbf{H z}$ \\
\hline Superpower & REBCO & 35 & $130.0 \mathrm{~mm}$ & $101.6 \mathrm{~mm}$ & $10.1 \mathrm{~m}$ & $20.18 \mathrm{Gauss}$ & $716.4 \mu \mathrm{H}, 2.39 \Omega$ \\
\hline
\end{tabular}

Figure 9 shows the $V$ - $I$ curves of the top and bottom coils. However, the $V-I$ curve for the top coil has the characteristics with a gentle curve, indicating a typical phenomenon for damaged coil. This gentle curve ${ }^{1}$ can be 
expressed as $A * I^{n}$ and must be subtracted from the original $V$ - $I$ curves in order to extract the intrinsic $V-I$ characteristics. With such alteration, the corrected $V-I$ curve for the top coil is obtained.

The maximum magnetic field on the REBCO rotor coil surface is measured as $2.02 \mathrm{mT} / \mathrm{A}$ in self-field, which means that at $133 \mathrm{~A}$, provided by manufacturer's data sheet, the field would be $0.264 \mathrm{~T}$. Similar to the previous analysis, the theoretical analysis value of $I_{c}$ is approximated by $\approx 56.8 \mathrm{~A}$, and the critical currents of $56 \mathrm{~A}$ and $57 \mathrm{~A}$ are obtained as the $I_{c_{-}} b o t$ and $I_{c_{-}} t o p$, respectively. The theoretical estimation of $\approx 56.8 \mathrm{~A}$ falls into the experimental value range of $56 \mathrm{~A}-57 \mathrm{~A}$.

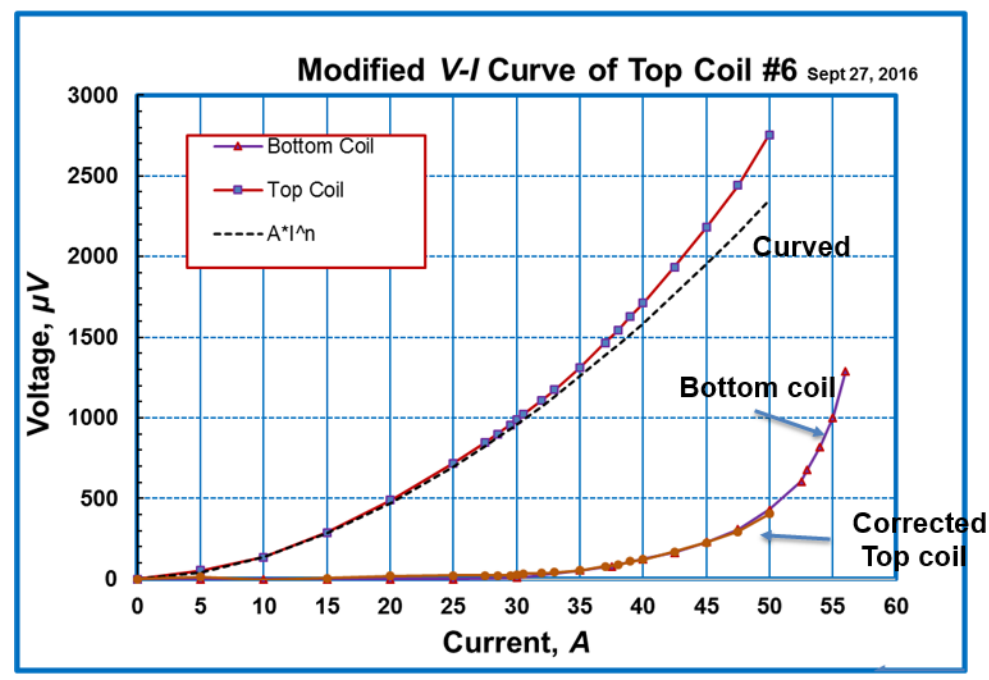

Figure 9. Plots of voltage vs. current for the REBCO rotor coil, showing the typical coil damage.
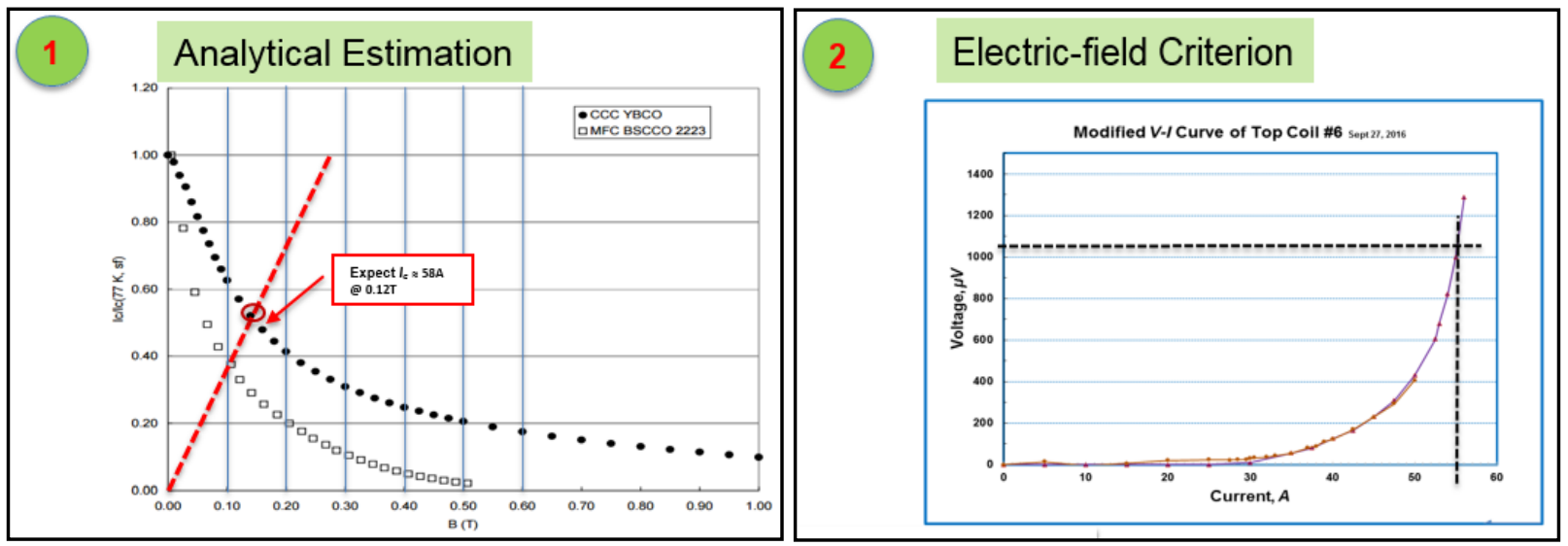

Figure 10. Critical current analyses of the double-layer YBCO rotor coil using (1) analytical estimation and (2) electric-field criterion.

Compared to the critical current of the YBCO coil, the performance degradation of the REBCO coil occurred. So, it is obvious that the REBCO coil has been damaged through the fabrication process and/or during the repeated cryogenic tests. Notice that $\mathrm{YBCO}$ and REBCO superconducting materials are multi-layer tapes that tend to delaminate the layers under some conditions such as repeated cyclic stresses, impact, and so on. This delamination can cause layers to separate, causing significant loss of coil performance. Hence, it is assumed that the coil might have been severely damaged due to the delamination and excessive soldering temperature. Thus, engineering judgement and skill and appropriate coil-winding fixture design are essential for successful coil fabrication and 
interconnection with soldered connections. Detailed remedies to minimize the HTS coil delamination will be discussed at the next publication.

\section{Quad-Stator REBCO Coil}
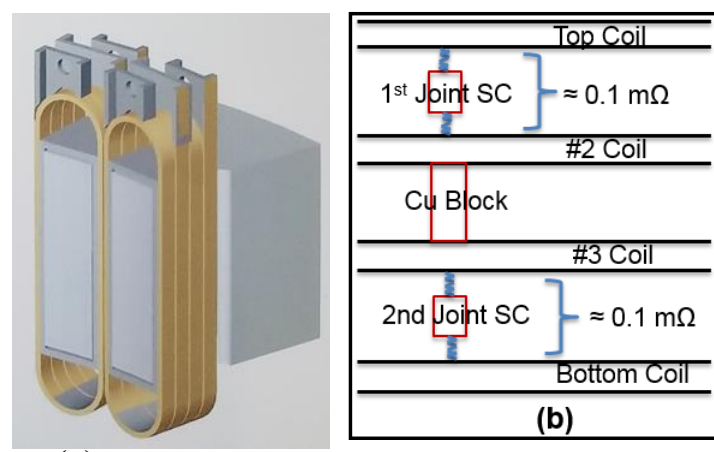

(a)

Figure 11. (a) Quad-stator coil and (b) schematic drawing of SC joint.
For the HTS stator coil winding, BSCCO and YBCO from American Superconductor cannot be used due to the minimum bending radius limit. Thus, only SuperPower REBCO tape was used to develop the prototype of four-coil packs that fit into the stator of existing superconducting motor test-bed as shown in Figure 11.a. There is a box-like structure of adaptor piece between the stator laminations and the coils. As illustrated in Figure 11.b, the first two coils are inner-connected by SC joint patches that have the resistance value of $0.1 \mathrm{~m} \Omega$. The second coil and the third coil are connected by a Cu block, and the third coil and the fourth coil are inner-connected by another SC joint patch that has also the resistance value of $0.1 \mathrm{~m} \Omega$. The coil dimension and technical data for the quad-stator coil are summarized in Table 5.

Consider the critical current analysis for the top coil only to assure the accurate setup of the apparatus. Since the total coil length between the two voltage taps is $1.2 \mathrm{~m}$, the critical voltage level of $120 \mu \mathrm{V}$ is chosen. Figure 12 shows the critical current is $\approx 77 \mathrm{~A}$, and the sharpness of superconducting-to-normal transition is $n=36.2$.

Table 5: Dimension and technical data for the quad stator REBCO coil.

\begin{tabular}{|l|l|l|l|l|l|l|l|}
\hline Manufacturer & Wire type & Turns/coil & O.D. & I.D. & Length/coil & Measured $\boldsymbol{B} / \boldsymbol{A}$ & $\boldsymbol{L}$ and $\boldsymbol{R} @ \mathbf{4 0 0} \mathbf{H z}$ \\
\hline Superpower & REBCO & 7 & $40.8 \mathrm{~mm}$ & $34.5 \mathrm{~mm}$ & $129.54 \mathrm{~cm}$ & 5.8 Gauss & $35.48 \mu \mathrm{H}, 0.556 \Omega$ \\
\hline
\end{tabular}

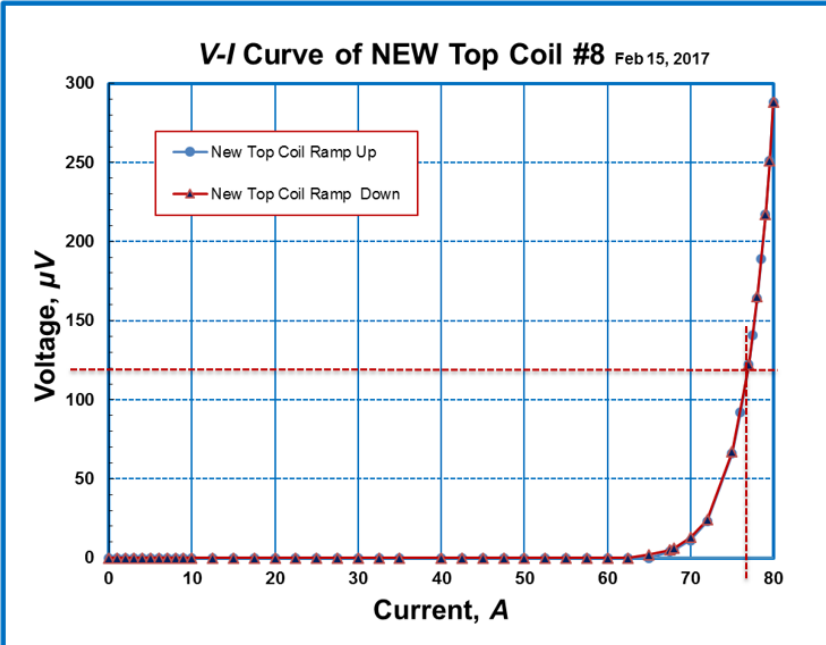

Figure 12. $V-I$ curve of the top coil only.

Figure 13 shows all four $V$ - $I$ curves of the four-coil packs. The bottom curve (red line with purple marker) shows the top coil (Coil 1) only, and the green curve with red marker shows the $V$ - $I$ curve of the Coils 1 and 2 . This curve includes the first $\mathrm{SC}$ joint resistance of $\approx 0.1$ $\mathrm{m} \Omega$ and the characteristics of Coils 1 and 2 . The red line with red marker shows the $V-I$ curve of Coil $1,1^{\text {st }} \mathrm{SC}$ joint, Coil 2, Cu block, Coil 3, and $2^{\text {nd }} \mathrm{SC}$ joint. Finally, the blue line shows the $V$-I curve of the whole coils between the voltage tap into the top coil and the voltage tap out of the bottom coil. Similar analysis done in the section III-B was

8

2017 American Institute of Aeronautics and Astronautics JPC 
conducted to subtract the resistance values to obtain the critical currents for each coil. $I_{c_{-}} l=43 \mathrm{~A}, I_{c_{-}} 2=27.5 \mathrm{~A}$, $I_{c_{-}} 3=25.5 \mathrm{~A}$, and $I_{c_{-}} 4=25.0$ were achieved.

Compared with the critical current value $\left(I_{c_{-}} l=77 \mathrm{~A}\right)$ of the top coil in Figure 12, the value of $I_{c_{-}} l=43$ A here, which had been measured again eight days later after some tests, is much lower. To investigate the cause of this coil performance degradation, a microscopy was utilized to examine the damaged coil surfaces. The microscopic images revealed that the coils were severely damaged due to the delamination. Thus, it is assumed that since the delamination process had been going on during the test, the performances for Coils 2, 3 and 4 have been reduced drastically since the Coil 1 measurement.

\section{E. REBCO Ironless Motor Coil}

For the rotor coil winding for the ironless motor, BSCCO and YBCO from American Superconductor cannot be used due to the minimum bending radius limit. Thus, only SuperPower REBCO tape was used to develop the prototype of skinny and long "race-track coil-pack (two interconnected coils in Fig. 14) for the superconducting motor test-bed. The coil dimension and technical data for the REBCO ironless rotor coil are summarized in Table 6.

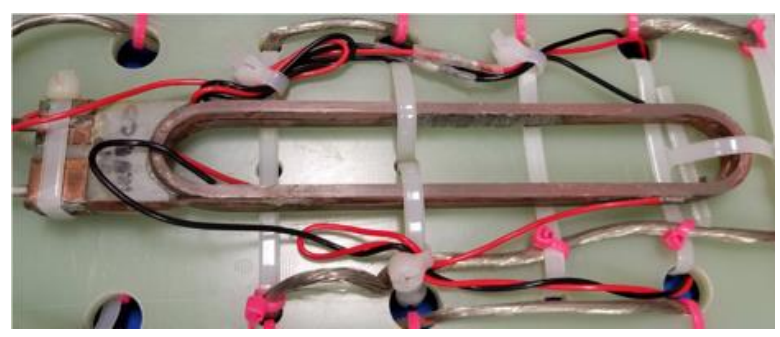

Figure 14. Rotor coil for the ironless motor.

The maximum magnetic field on the REBCO ironless motor coil surface was measured as $1.285 \mathrm{mT} / \mathrm{A}$, which means that at $133 \mathrm{~A}$, provided by manufacturer's data sheet, the field would be $0.170 \mathrm{~T}$. Like the previous analysis, the theoretical analysis value of $I_{c}$ is approximated by $\approx 78$ A. For the electric-field criterion

analysis in Figure 15, the critical current for the top coil (purple curve) can be obtained as $I_{c}=54 \mathrm{~A}$, which is much smaller than that of the theoretical estimation.

Further, the $V$-I curves for the bottom coil and whole coil are getting worse. The microscopic investigation revealed that the REBCO coils got severely damaged. Very much like the previous REBCO quad-coil case, it is assumed that since the delamination process had been going on during the test, the performances for the bottom coil and whole coils are getting worse than that of the top coil, which was measured first.

Table 6: Dimension and technical data for the REBCO rotor coil for the ironless motor.

\begin{tabular}{|l|l|l|l|l|l|l|l|}
\hline Manufacturer & Wire type & Turns/coil & O.D. & I.D. & Length/coil & Measured $\boldsymbol{B} / \boldsymbol{A}$ & $\boldsymbol{L}$ and $\boldsymbol{R} @ \mathbf{4 0 0} \mathbf{H z}$ \\
\hline SuperPower & REBCO & 22 & $40.8 \mathrm{~mm}$ & $34.5 \mathrm{~mm}$ & $4.09 \mathrm{~m}$ & 12.85 Gauss & $251.9 \mu \mathrm{H}, 1.58 \Omega$ \\
\hline
\end{tabular}

\section{Summary}

In this paper, we have presented 1) DC critical current $\left(I_{c}\right)$ measurements on three compact "race-track" prototypes of HTS rotor coil-packs, one stator coil-pack, and one ironless motor coil-pack at liquid nitrogen temperature $(77 \mathrm{~K})$ in self-field, 2) sharpness ( $n$ value) of the super-to-normal transition, and 3) standard procedures for coil design, fabrication, micro-volt measurement, and cryogenic testing, current control, and data acquisition technique. Measured critical current data based on the electric-field criterion $\left(E_{c}\right)$ was compared to the theoretical prediction, and they agreed well within a reasonable margin. However, some REBCO coils have been severely damaged due to the delamination during the repeated tests and excessive soldering temperature during 
the fabrication process, causing drastic performance degradation. Thus, new coil winding technique, cryogenic coating, and engineering judgement must be taken into account for future test.

Established knowledges at $77 \mathrm{~K}$ here such as lessons learned, data analysis skills, standard procedures for coil design, fabrication, and measurement technique will be used to assess the feasibility of using HTS wire materials in future cryogenically-cooled fully superconducting air breathing EAP systems. Also, it would be a guide for the low temperature $(20 \mathrm{~K})$ superconducting coil $\left(\mathrm{MgB}_{2}\right)$ test bed, which is under development at GRC.

\section{Acknowledgments}

This work was in full support of the Electrified Aircraft Propulsion (EAP) under Advanced Air Transportation Technology (AATT) Project at NASA Glenn Research Center. The authors thank to Mr. Ben Ebihara at Vantage Partners Co. for his great efforts in designing and fabricating the HTS coils and superconducting motor assemblies.

\section{References}

${ }^{1}$ Ekin, Jack W., Experimental Techniques for Low-Temperature Measurements: 1906-1921, Oxford University Press, ISBN 978-0-19-857054-7, Great Clarendon Street, Oxford ox2 6 DP, reprinted in 2015.

${ }^{2}$ Aized, D., Haddad, J. W., and Joshi, C. H., "Comparing the Accuracy of Critical-Current Measurements Using the VoltageCurrent Simulator,” IEEE Transactions on Magnetics, Vol. 30, No. 4, July 1994.

${ }^{3}$ Horng, Lance, and Chen, K., "Voltage Criterion of Transport Critical Current Density for a Bi-based Superconducting Tape," The Physical Society of the Republic of China, Vol. 36, No. 2-II, April 1998.

${ }^{4}$ Ghosh, Arup K., "V-I Transition and $n$-value of Multifilamentary LTS and HTS Wires and Cables," ICMC'03 Topical Conference, Enschede, Netherland, May 25-28, 2003.

${ }^{5}$ Malozemoff, A. P., Verebelyi, D. T., Fleshler, S, Aized, D., and Yu, D., "HTS Wire: Status and Prospects," published in Physica C, Vol. 383 (15 April 2003), Proceedings of ICMC 2002, Xi' an China, June 16-20, 2002, pp. 424-430.

${ }^{6}$ Fleshler, S., Fee, M., Spreafico, S., and Malozeoff, A. P., Advances in Superconductivity XII, Yamashita, T., and Tanabe, K. (Springer, Tokyo, 2000), pp. 625-630. 